\title{
Smartphone-based Oxygen Imaging Sensor and Application to BOD sensor
}

\author{
Masayasu Suzuki, Daisuke Shimizu, Yuki Horiuchi, Yasunori Iribe \\ University of Toyama, Toyama, Toyama 930-8555, Japan \\ suzukimy@eng.u-toyama.ac.jp
}

\begin{abstract}
:
Fluorescent oxygen sensor image was obtained by using a CMOS camera in an Android OS smartphone. The obtained image was analyzed by using I_J mobile, an image analysis software for Android OS. Difference of oxygen concentration could be detected by using a red color image obtained by this system. White LED backlight module for fluorescence probe excitation could function well also with AAA cell batteries, or button batteries. By putting an yeast immobilized membrane on the oxygen sensor film, BOD could be detected by the difference of fluorescence intensity. The present simple and low cost sensor is suitable for field monitoring in developing countries.
\end{abstract}

Key words: smartphone, oxygen sensor, BOD sensor, fluorescence, field monitoring

\section{Introduction}

Environmental pollution in developing countries is a serious social problem. To manage this problem, a regular environmental monitoring is needed, but currently, there is a lacking of measuring tools making the evaluation of pollution very difficult. Therefore, with the development of a low-cost environmental monitoring biosensor, a more regular in-situ monitoring can be done which will hopefully resolve the problem of pollution of river or lake water. We have already developed a simple and low cost BOD sensor based on oxygensensitive fluorescent dye [1]. But the instruments for fluorescence measurement are still expensive and not suitable for field use. We focused on smartphones. Nowadays, smartphones are widely used even in developing countries. Smartphones are now similar to miniature computers with high-quality camera, and there have been many publications on the use of smartphones as bioanalytical devices [2]. In this study, smartphone is used as a detector for optical oxygen imaging sensor, and applied to BOD sensor.

\section{Materials and Methods}

\section{Sensor chip}

Polyvinyl alcohol $0.6 \mathrm{~g} / \mathrm{l}$ was dissolved in $5 \mathrm{mM}$ MOPS buffer ( $\mathrm{pH} 7.0)$. Ruthenium complex dye (dichloro tris (1,10-phenanthroline) ruthenium (II)), $1 \mathrm{~g} / \mathrm{l}$ with the above solution and equal volume of $5 \%$ nafion solution was completely mixed. The prepared dye solution, $2 \mathrm{ml}$ was dropped onto a diamond like carbon coated glass slide which has $\mathrm{SO}_{3}$ groups on the surface to coat the whole surface area of the glass slides. The baker's yeasts (Saccharomyces cerevisiae ) were cultured at 35C on yeast-extract peptone dextrose (YPD) agar media. Yeasts were washed twice with PBS, and its suspension was mixed with photocrosslinkable polyvinyl alcohol (PVA-SbQ). The mixture was spread and dried at $4 \mathrm{C}$. Photocrosslinking was done with fluorescence lamp.

\section{Smartphone-based flurorescence imaging unit}

The structure and photo of prepared smartphone-based fluorescence imaging unit were shown in Fig.1 and 2, respectively. Android OS smartphone Experia Z5 (SONY) was used in this study. This smartphone has a CMOS camera Exmor RSW for mobile (ca. 23 mega pixels). A clip lens for smartphone was set on it. For excitation and emission filters, Wratten No.4 (450 $\pm 40 \mathrm{~nm}$, Kodak) and Wratten No.12 (500nm , Kodak) were used. White LED backlight module (\#1622, Adafruit Industries) has the same size as a glass slide. Light shielding unit was prepared by using a 3D printer

\section{Results and Discussion}

\section{Response to oxygen}

Fluorescence image was obtained by using 


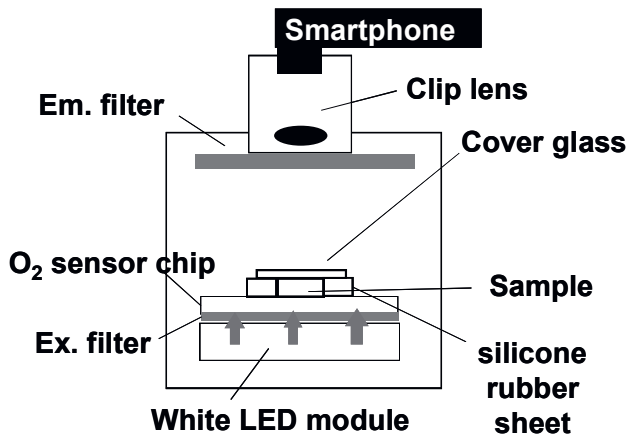

Fig.1 Smartphone-based fluorescence imaging unit

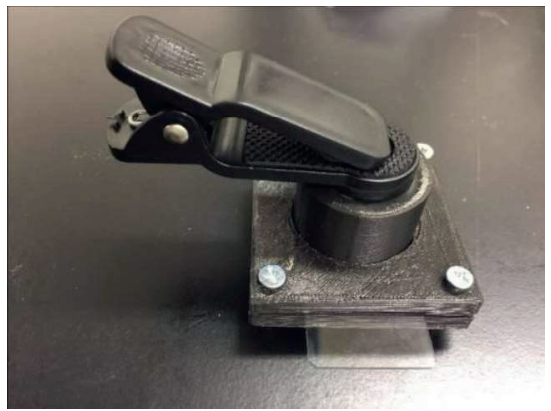

Fig. 2 Imaging unit with a clip lens

CMOS camera in Xperia Z5. Fluorescence intensity of obtained fluorescence image was measured by using I J mobile (Android OS version of image $\mathrm{J}$ ). The color image was separated into R, G and B components. Only the red component showed strong dependency on oxygen concentration (Fig.3).

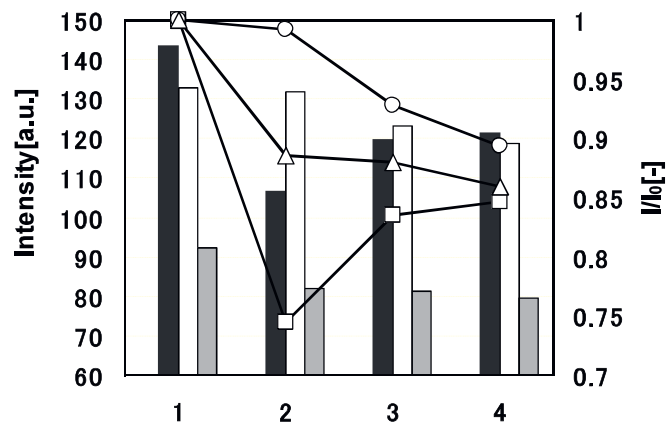

Fig.3 RGB intensity of fluorescent oxygen sensor in different oxygen concentrations.

Red (dark grey columns, $\square$ ), Green(white columns, $\bigcirc$ ), $(R+G+B) / 3$ (pale grey colmns, $\triangle$ ). Relative responses normazed by the response to $5 \% \mathrm{Na}_{2} \mathrm{SO}_{3}$ were indicated with $\square, O, \triangle$ )

Samples:(1)5\% $\mathrm{Na}_{2} \mathrm{SO}_{3}$, (2)oxygen aerated water (3) distilled water, (4)nitrogen aerated water.

\section{Electric Supply for LED Light Source}

The white LED module used in this study works with DC 3V. Firstly two AAA cell batteries were used as an electric source. Fluorescence intensity for $5 \% \quad \mathrm{Na}_{2} \mathrm{SO}_{3}$ became $85 \%$ compared with conventional DC supply, but relative responses to oxygen concentration was the same (Fig.4). In the same way, the smaller button-shaped batteries, CR2032(3V) and LR44(1.5V x 2) were used as an electric source. Fluorescence intensity became smaller, but relative response to oxygen concentration was the same. The batteries for LED were set in the bottom part of sensor unit (Fig.5).

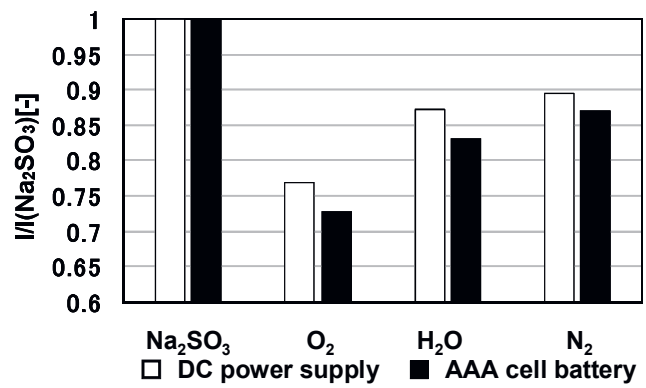

Fig. 4 Oxygen measurement using $A A A$ cell batteries

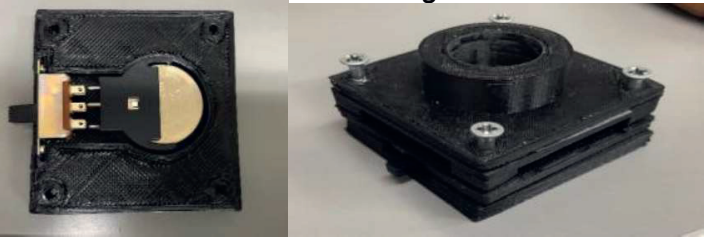

Fig. 5 A case for CR2032 and an imaging unit

\section{BOD sensor}

Immobilized yeast film was put on an oxygen sensor chip and BOD standard solutions ( 0 and $220 \mathrm{mg} / \mathrm{l})$ prepared with a phosphate buffer were measured alternately. Ten percentage increase of fluorescence intensity could be observed in BOD solution.

$220 \mathrm{mg} / \mathrm{l} \mathrm{BOD}$

(glucose and glutamate, $150 \mathrm{mg} / \mathrm{l}$ each) Omg/l BOD (PBS)

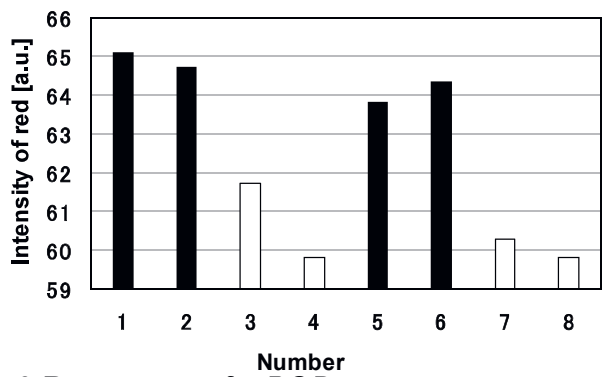

Fig. 6 Responses of a $B O D$ sensor

\section{References}

[1] M.A.Kashem, M. Suzuki, K.Kimoto, Y.Iribe, An optical biochemical oxygen demand biosensor chip for environmental monitoring, Sensors and Actuators B, 221, 1594-1600 (2015); doi: 10.1016/j.snb.2015.07.119

[2] A. Roda et al., Smartphone-Based Biosensors:A Critical Review and Perspectives, Trends in Analytical Chemistry 79, 317-325 (2016); doi: 10.1016/j.trac.2015.10.019 